\title{
Research Progress on TCM Treatment of Behcet's Disease
}

Jia Shi

The First Affiliated Hospital of Tianjin University of Traditional Chinese Medicine, Tianjin 300380, China

\begin{abstract}
Behcet's disease is a vasculitis disease involving multiple systems, and its pathogenesis is still unclear. The paper discusses the research progress on the treatment of Behcet's disease in traditional Chinese medicine and integrated traditional Chinese and Western medicine. The clinical efficacy of integrated traditional Chinese and western medicine in the treatment of Behcet's disease is significantly better than that of Western medicine alone, which is worthy of further research.
\end{abstract}

Keywords: Behcet's Disease; Etiology and Pathogenesis; Traditional Chineese Medicine Treatment; Chinese and Western Medicine Treatment; Overview

Publication date: January, 2021

Publication online: 31 January, 2021

"Corresponding author: Jia Shi, fx7777777sj@163. com

Behcet's disease (BD), also known as Behcet's syndrome, mouth-eye-genitals triad syndrome, and Silk Road disease, is a chronic autoimmune disease with unidentified causes and vasculitis as its basic pathological changes ${ }^{[1]}$. The clinical manifestations of this disease are complex and diverse. In addition to the characteristic manifestations such as recurrent oral ulcers, genital ulcers, ophthalmia and skin damage, it can also invade joints, nervous system, digestive tracts, cardiovascular and other organs. The prognosis of most patients is fair, but the prognosis of most patients with central nervous system, eye and large blood vessels implications is often poor. There is no cure for this disease in modern medicine. The first-line treatments mainly include thalidomide, colchicine, glucocorticoids, immunosuppressive agents, and biological agents etc. Not only is drug withdrawal prone to relapse, but the toxic sideeffects of drugs often hurt patients greatly. Traditional medicine has unique and certain advantages in the treatment of this disease. The author now summarizes the clinical research progress on Chinese medicine in the treatment of BD in recent years.

\section{Etiology and Pathogenesis}

BD belongs to the category of "fox possession disease" in traditional Chinese medicine. It was first seen in Zhongjing's "Jingui Yaolue • Pulse Syndrome Differentiation and Treatment of Lily, Fox Possession and Yin-Yang Toxic Diseases": "Fox possession is a disease like typhoid fever... ulcers in the throat is known as possession, ulcers in the private part is known as fox", "the patient's pulse is few, no heat, slight annoyance, silent and want to lie down... the eyes are red like dove eyes", the symptoms of "ulcers up and below, eyes red like dove eyes" mentioned here is very similar to the characteristic clinical manifestations of this disease such as oral ulcers, external genital ulcers, and ophthalmia etc. Since then, physicians of the past dynasties also elaborated on the etiology and pathogenesis of fox possession disease. Sui Dynasty's Chao Yuanfang stated in the "General Treatise on Causes and Manifestations of All Diseases (Zhubing Yuanhou Lun)" that the disease was "totally caused by dampness and toxins", "or it may be a result of typhoid fever"; Tang Dynasty's Sun Simiao's "Qianjin Yaofang” pointed out that this disease is the result of the accumulation of "warm toxins and evil qi", which established the theory of "damp heat" as the basic pathogenesis of the fox possession disease.

On the basis of inheriting the theories of the ancients, modern doctors have further deepened their understanding and research on the disease after a great deal of clinical practice. Yue Meizhong, a master of the school of Chinese classic herbal 
formula in modern times, once said: "When warm toxin febrile diseases were treated inappropriately, evil and toxins that are unable to escape will seek their way out and turn into a severe disease, that is, the fox possession disease"; through the summarization and analysis on the treatment of the disease by many doctors in modern times, Yan Zhen$\mathrm{bo}^{[2]}$ opined that the external damp hot toxic evil invaded the body at the beginning of the onset of the disease, and in the later stage spleen, stomach, and kidney yin are exhausted, leading to the functional disorders of the viscera; or if the body is depleted, and the yin, yang, qi and blood of the viscera are imbalanced, it will result in the generation of "dampheat toxins and evil", which are considered to be an important internal cause of the disease; from the perspective of "excess qi result in fire" proposed by Zhu Danxi, one of the four masters of the Jin and Yuan Dynasties, $\mathrm{Li} \mathrm{Xia}^{[3]}$ opined that both solid and vain fires can rise and scorch, so we find sores in the mouth and itching in the eyes; if the hot toxic evil hides in the body and cannot be gasified, it will find a way out, and lead to ulceration in the vulva with the descent of damp evil; if it invades the skin and flows into the joints, it will cause skin erythema nodules and arthralgia. As the disease progresses, the strengthening evil will hurt the normal qi, resulting in damage to multiple systems such as, kidneys, blood vessels, nerves, etc. Therefore, "hot and toxic evil" must not be ignored in the incidence and development of $\mathrm{BD}$; from the perspective of modern medicine, Chen Yong preliminarily discussed and explained the mechanism of the onset of BD combining Chinese and Western medicine. He identified "worm toxins" as pathogenic microorganisms, comprehended the genetic susceptibility of HLA-B51 as the concept of innate endowment insufficiency in traditional Chinese medicine, and provided preliminary modern medical understanding on the concepts of deficiency, blood stasis, damp-heat in the pathogenesis of BD.

\section{Traditional Chinese Medicine Treatment}

\subsection{Syndrome Differentiation and Treatment}

$\mathrm{Su} \mathrm{Li}$ believes that the main etiology and pathogenesis of BD are "spleen failure, liver meridian damp heat and blood stasis toxins", and formulated the treatment principle of "prioritize clearing heat and removing dampness, resolving blood stasis and detoxification, and strengthening normal qi throughout the course", and devised treatments for BD according to its symptoms, manifestations and stages: in the acute stage, clearing heat, removing dampness, resolving blood stasis and detoxification are the main tasks, supplemented with qi replenishing and yin nourishing medicines to strengthen the body; in the chronic stable stage, the main tasks are replenishing qi and nourishing yin, supplemented with heat clearing, dampness removal and detoxification. Li Guangyao believes that $\mathrm{BD}$ often presents an alternating process of repeated relapses and remissions. According to the different syndromes, symptoms, organs involved and hormone use, the "disease-syndrome-symptom combination" should be used to treat this disease: Oropharyngeal ulcers are mostly caused by heat in the lungs and stomach, treat with Gancao Xiexin Decoction as the main prescription; skin lesions are treated based on phlegm and blood stasis, and Simiao Yongan Decoction is the main prescription; eye and vulvar ulcers are treated based on the liver, and the prescription is modified Qinghao Biejia Decoction; gastrointestinal ulcers are treated based on the spleen, and it is suitable to use both cold and warm prescriptions for treatment together with Wumei Pills. Wang Zhen believes that the site of $\mathrm{BD}$ is mainly in the pulse and often accompanied by mental symptoms such as insomnia, dreaminess, forgetfulness, irritability, etc., so he proposed to treat Behcet's disease based on the heart, starting from both the aspects of "blood and pulse" and "mental": on the one hand, the treatment principles of clearing heart and calming nerves, nourishing yin and clearing heat, replenishing qi and blood, and promoting blood circulation to remove blood stasis are adopted according to different syndromes; on the other hand, $\mathrm{BD}$ is a physical and mental disease, it is necessary to pay attention to the mental state of patients, strengthen mental health counseling, and targeted psychotherapy should be given when required.

\subsection{Special and Proven Prescriptions}

$\mathrm{Xu}$ Yubo and others applied the Sanwu Huangqin Decoction recorded in "Essential Formulas for Emergencies Worth a Thousand Pieces of Gold (Beiji Qianjin Yaofang)" to treat 30 patients with Behcet's disease. As a result, 20 cases were cured, 9 cases improved, 1 case was ineffective, and the total efficacy was $96.67 \%$. Shi Haijun and others 
randomly divided 40 patients with $\mathrm{BD}$ into 2 groups: 20 patients in the experimental group were treated with Baisai Buxie Granules. The whole prescription consisted of Oldenlandia diffusa, barbed skullcap, Danpi, Danshen, liquor rhubarb, Adenophora, lilyturf, schisandra, evodia, dried ginger, licorice, roasted licorice, cornus, sarsaparilla, shrubby sophora, Danggui, coptis, skullcap, phellodendron, fried atractylodes, etc.; while 20 cases in the control group were treated with thalidomide. Results show that the total clinical efficacy $(90 \%)$ of the experimental group was significantly higher than that of the control group (75\%), and the ESR and CRP of the experimental group were significantly better than the control group. The difference between the two groups was statistically significant $(P<0.05)$. The recurrence rate of patients in the experimental group was also lower than that of the control group $(P<0.05)$. Zhang Zhirui et al. randomly divided $100 \mathrm{BD}$ patients into a experimental group and a control group with 50 cases each: the experimental group was given Gancao Xiexin Decoction modified with San-huang (Coptis (Huanglian), Astragalus (Huangqi), Skullcap (Huangqin)) treatment; the control group was given oral prednisone treatment. Results show that the total efficacy $(82 \%)$ of the experimental group was significantly higher than that of the control group (72\%), and the comparison of the efficacy between the two groups was statistically significant $(P<0.05)$.

\subsection{Integrated Traditional Chinese and Western Medicine}

Li Huaying et al. randomly divided 114 patients with Behcet's disease into 2 groups: 57 cases in the control group were treated with Western medicines such as prednisone and levamisole; 57 cases in the experimental group were treated with Chixiaodou Danggui San on the basis of the Western medicine treatment in the control group . Results: The total efficacy was $78.9 \%$ in the control group and $94.7 \%$ in the experimental group. The difference between the groups was statistically significant $(P<0.05)$, and the fibrinolytic system indexes of the experimental group were all lower than control group $(P<0.05)$. Qu Huanru et al. ${ }^{[4]}$ randomly divided 40 cases of BD patients with syndromes of qi deficiency and blood stasis into two groups: 20 cases in the control group were treated with thalidomide, and 20 cases in the experimental group were treated with Yiqi
Jiedu Quyu Decoction (consisting of astragalus, raw rehmannia, white turmeric, skullcap, smilax, chinese peashrub root, rhubarb, raw licorice, and roasted licorice) on the basis of the control group. Result shows that the total efficacy was $95.00 \%$ in the experimental group and $85.00 \%$ in the control group. The difference between the groups was statistically significant $(P<0.05)$; and the oral ulcer intermittent time in the experimental group was longer than the control group after treatment $(P<0.05)$, the ESR was lower than the control group $(P<0.05)$; the incidence of adverse reactions in the experimental group $(5.0 \%)$ was significantly lower than that of the control group (35.0\%). Comparing the two groups, the difference was statistically significant $(P<0.05)$. Wang Yong et al. divided 50 patients with Behçet's syndrome into a integrated Chinese-Western medicine group and a Western medicine group with 25 cases each. The Western medicine group was treated with Iramud tablets and the Chinese-Western medicine group was given a self-made modified prescription for resolving phlegm and removing blood stasis in conjunction with the control group treatment. The prescription is composed of pinellia rhizome, Danggui, rehmannia glutinosa, Chinese tuckahoe, fried peach kernel, red peony root, Chuanxiong, tangerine peel, licorice, dayflower, Xiaojin Pian, Euchresta japonica, Tianmingjing, arrowshaped tinospora root, raw terminalia fruit, river bulrush, and white turmeric. Results show that the clinical efficacy of the ChineseWestern medicine group (total efficacy $95.30 \%$ ) was significantly better than that of the Western medicine group (total efficacy $87 \%$ ), and the difference was statistically significant $(P<0.05)$; and the CRP and IgA levels in the Chinese-Western medicine group were lower than the Western medicine group, the difference was statistically significant $(P<0.05)$.

\section{Conclusions}

This paper summarizes the literature on the treatment of BD with traditional Chinese medicine in recent years. It was found that traditional Chinese medicine has a deep understanding of this disease. Chinese medicine advocates syndrome differentiation and treatment. It has a unique set of diagnosis and treatment methods for this disease and has significant clinical effects. It is significantly better than pure Western medicine treatment and is worthy of 
further research. However, the above studies are not conducive to clinical promotion due to the lack of large samples, in-depth study of the mechanisms of Chinese medicine, and many doctors' using self-made prescriptions etc. If clinical research can be carried out under scientific and rigorous experimental design, and learn from modern advanced scientific research results, it will further provide a more objective and powerful basis for the treatment of BD by Chinese medicine, and provide standardized Chinese medicine diagnosis and treatment schemes for better promotion and application of Chinese medicine.

\section{References}

[1] Chen QQ, Li JX, Fu XL. Research progress on genetics of Behcet's disease [J]. Chinese Journal of Rheumatology, 2017(1).

[2] Yan ZB. Research Progress on the Etiology and Pathogenesis of Behcet's Disease in Traditional Chinese Medicine [J]. World Latest Medicine (Electronic Version), 2018, 18(6): 117-117.

[3] Li X, Zhang HY, Bai C, Zhang LT. Discussion of Behcet disease according to excessive Qi causing fire [J]. Clinical Journal of Traditional Chinese Medicine (6):869-870.

[4] Qu HR, XI SJ, CAO ZY, et al. Clinical observation of "Yiqi Jiedu Quyu Decoction" and thalidomide in treating Behcet's disease [J]. Shanghai Journal of Traditional Chinese Medicine, 2016(5): 48-50. 